\title{
Modeling of crossbred cattle growth, comparison between cubic and piecewise random regression models
}

\author{
H.R. Mirzaei ${ }^{1}$, W.S. Pitchford ${ }^{2}$ and A.P. Verbyla ${ }^{3}$ \\ ${ }^{1}$ Department of Animal Science, Zabol University, Zabol, Iran \\ ${ }^{2}$ Animal and Veterinary Sciences, University of Adelaide, Adelaide, Australia \\ ${ }^{3}$ School of Agriculture, Food and Wine, Waite Campus, \\ University of Adelaide, Adelaide, Australia \\ Corresponding author: H.R. Mirzaei \\ E-mail: president@imamreza.ac.ir/h.mirzaei9@gmail.com
}

Genet. Mol. Res. 10 (3): 2230-2244 (2011)

Received January 31, 2011

Accepted May 25, 2011

Published September 27, 2011

DOI http://dx.doi.org/10.4238/vol10-3gmr1302

\begin{abstract}
Two analyses, cubic and piecewise random regression, were conducted to model growth of crossbred cattle from birth to about two years of age, investigating the ability of a piecewise procedure to fit growth traits without the complications of the cubic model. During a four-year period (1994-1997) of the Australian "Southern Crossbreeding Project", mature Hereford cows $(\mathrm{N}=581)$ were mated to 97 sires of Angus, Belgian Blue, Hereford, Jersey, Limousin, South Devon, and Wagyu breeds, resulting in 1141 steers and heifers born over four years. Data included 13 (for steers) and eight (for heifers) live body weight measurements, made approximately every 50 days from birth until slaughter. The mixed model included fixed effects of sex, sire breed, age (linear, quadratic and cubic), and their interactions between sex and sire breed with age. Random effects were sire, dam, management (birth location, year, post-weaning groups), and permanent environmental effects and for each of these when possible, their interactions with linear, quadratic and cubic growth. In both models, body weights of all breeds
\end{abstract}


increased over pre-weaning period, held fairly steady (slightly flattening) over the dry season then increased again towards the end of the feedlot period. The number of estimated parameters for the cubic model was 22 while for the piecewise model it was 32 . It was concluded that the piecewise model was very similar to the cubic model in the fit to the data; with the piecewise model being marginally better. The piecewise model seems to fit the data better at the end of the growth period.

Key words: Crossbred cattle; Growth; Cubic; Random regression; Piecewise

\section{INTRODUCTION}

In the beef industry, the optimisation of cattle production systems including the evaluation of alternative management and marketing strategies for individual breeder, backgrounder and finisher operations to get most favourable end products requires knowledge of the variation in body weights during the growth path. Individual animals have their own growth path. Several difficulties are encountered in fitting non-linear growth models (Schinckel et al., 2005; Forni et al., 2009; Speidel et al., 2010). Shortcomings include fixed inflection points, assumption of a monotonic increase in size from origin to asymptote and the inestimable errors in various adjustment factors, causing growth to be under or over estimated (Brown et al., 1976; Fitzhugh Jr., 1976). Bio-economically, there are obvious distinctions between different stages during growth path: pre- and post-weaning growth; consequently, there has been great interest in their characteristics. The cubic model for calf change, however, does not readily lend itself to exploring issues of this kind. Piecewise models for animal growth provide a means of dividing successive ages into meaningful segments, and capturing key features of change in each segment. In other words, piecewise linear growth models are attractive when focusing on the comparison of growth curves in two different periods, or investigating whether the predictors of growth change over specific periods. Information, using a piecewise linear model in beef cattle growth, is very limited. Warren et al. (1980) described the method for segmented line regression, and explained it with an example using data from Hereford females.

The main objective of the study was to provide a view of two analyses, cubic and piecewise random regression, investigating the ability of a piecewise linear regression procedure (Hodson, 1966) to fit growth traits without the difficulties mentioned for the cubic model. Thus, it was focused on the fundamental statistical and then biological similarities and differences of the two models to address: Whether the piecewise model fits the data better than the cubic model or not? and how?

\section{MATERIAL AND METHODS}

\section{Animals and management}

The animals from the "Southern Crossbreeding Project" have been used for this study. The Southern Crossbreeding Project was designed to characterise between and within breed variations with the aim of improving utilisation of existing breeds for meeting a range of 
market specifications in southern Australia (Pitchford et al., 2006). Purebred Hereford cows (581) were mated to semen of sire breeds Angus (11 sires), Belgian Blue (16 sires), Hereford (10 sires), Jersey (12 sires), Limousin (16 sires), South Devon (15 sires), and Wagyu (17 sires). There were generally $12-15$ progeny per sire, with an average of 13 calves per sire and 14 sires per breed. The project comprised 1141 of the heifers (females) and steers (castrated males) born in autumn (average birth date 3rd April) at two locations ("Struan" near Naracoorte and "Wandilo" near Mount Gambier in the south east of South Australia), over a 4-year period (1994 to 1997). Calves were weaned in summer (mid December-early January) at 250 to 300 days of age, each year, i.e., in most years the last weight represented a weaning weight. At weaning, all calves born at Wandilo were transferred to Struan. Calves stayed with their dams on pasture until weaning and calves were grown until 12 to 18 months of age and then transported to a commercial feedlot except the 1997 steers which, after a good pasture season in 1998, reached marketable weight without requiring grain finishing (Pitchford et al., 2006).

\section{Live body weights (growth)}

Live body weights (unfasted) consisted of 13 measurements for steers and 8 measurements for heifers at approximately every 50 days from birth until slaughter. Table 1 shows summary statistics of the weight-age array from birth to slaughter for steers and heifers. The means were averaged over all four years. The standard deviation for live weight of both heifers and steers increased from the first to the last weighing (Table 1). To overcome this heterogeneous variance, the use of the natural logs of the body weights rather than the original body weights seemed sensible. Thus,

$$
\begin{aligned}
& \mathrm{y}_{\mathrm{t}}=\ln (\mathrm{BW})_{\mathrm{t}} \sim \mathrm{N}\left(\mu_{\mathrm{t}}, \sigma_{\mathrm{t}}^{2}\right) \\
& \mathrm{BW}=\exp \left(\mathrm{y}_{\mathrm{t}}\right)
\end{aligned}
$$

\begin{tabular}{|c|c|c|c|c|c|c|c|}
\hline \multicolumn{4}{|c|}{ Heifers } & \multicolumn{4}{|c|}{ Steers } \\
\hline Mean (days) & $\mathrm{SD}$ & Mean (kg) & SD & Mean (days) & SD & Mean (kg) & $\mathrm{SD}$ \\
\hline 0 & 0 & 36.54 & 6 & 0 & 0 & 39.01 & 6 \\
\hline 75 & 22 & 93.32 & 23 & 75 & 22 & 98.10 & 24 \\
\hline 125 & 21 & 124.22 & 29 & 125 & 22 & 130.93 & 30 \\
\hline 175 & 19 & 17.52 & 36 & 175 & 20 & 183.02 & 38 \\
\hline 230 & 26 & 239.84 & 43 & 230 & 27 & 256.00 & 44 \\
\hline 280 & 28 & 277.66 & 40 & 280 & 29 & 295.61 & 40 \\
\hline 330 & 32 & 296.44 & 50 & 330 & 33 & 303.38 & 42 \\
\hline 415 & 20 & 333.50 & 48 & 387 & 32 & 329.40 & 37 \\
\hline- & - & - & - & 438 & 36 & 349.23 & 40 \\
\hline- & - & - & - & 482 & 27 & 353.09 & 48 \\
\hline- & - & - & - & 545 & 36 & 414.13 & 70 \\
\hline- & - & - & - & 593 & 44 & 481.82 & 92 \\
\hline- & - & - & - & 630 & 65 & 532.61 & 110 \\
\hline
\end{tabular}

where $y$ is normally distributed with mean $\mu$ and standard deviation $\sigma$.

Number of observations $=1141$.

Age was centered (mean subtracted) and scaled (from days to years). This was done for both numerical reasons and for prediction. In the former case changes in one predictor 
could be gauged by setting others at their mean, i.e., at the new origin for centered age and in the later, (co)variance components were more easily estimated because they were larger.

\section{Statistical analysis}

\section{Random regression analysis}

Growth of animals is characterized by a) tracking: that is individual animals have their own growth path, and hence these paths vary between animals, b) increased variation as animals grow: this is related to tracking in that some animals have a stronger growth rate than others. The variation in path and in spread over time can be captured by random regression model (though not always completely). This model accommodates correlation, increasing variances and allows animal-specific growth paths. This is achieved by allowing regression coefficients for weight on time to be random effects across individuals, which are correlated within individuals. Thus, an animal is viewed as being sampled via these correlated random regression coefficients.

\section{Cubic model}

The growth models considered in this paper are the mixed models. The response variable is log-body weight. This is traditionally the scale on which weight is analyzed, mainly because of the "multiplication" nature of growth. The added benefit is that the heterogeneity in variation is reduced. Finally, the implicit assumption is that the random variation has a log-normal distribution, and various properties can be used to provide results on the original weight scale. Let $y$ be the vector of log-body weights for all animals at all measurement times.

The mixed model is $\mathrm{y}=\mathrm{X} \tau+\mathrm{Zu}+\mathrm{e}$, where $\tau$ is the vector of fixed effects; $u$ is the vector of random effects; $e$ is the vector of residual errors.

The matrices $\mathrm{X}$ and $\mathrm{Z}$ contain covariate values and indicators (factors) specific to each animal and sometimes to each measurement time. They extract the appropriate elements of $\tau$ and $u$ for each animal and time. General assumptions are that $u \sim \mathrm{N}(0, \mathrm{G})$ and $e \sim \mathrm{N}(0, \mathrm{R})$ for some (co)variance matrices $\mathrm{G}$ and $\mathrm{R}$. In addition, $u$ and $e$ are assumed independent. The form for $\mathrm{G}$ and $\mathrm{R}$ depends on the actual model. We turn to the specification of the growth models considered in this chapter. Note that under the above general mixed model,

$$
\mathrm{E}(\mathrm{y})=\mathrm{X} \tau=\mu \text {, say and } \operatorname{var}(\mathrm{y})=\mathrm{R}+\mathrm{ZGZ}^{\mathrm{T}}
$$

The mean $\mu$ depends on the fixed effects included in the model. The parameters associated with the fixed effects are given by $\tau$. The model considered was the sire model. The fixed effects involve breed, sex, age (time), and interactions between breed and sex with age. As the cubic polynomial forms the basis of time or age growth trends, breeds and sexes are allowed to differ in their cubic growth path. Let $i$ denote breed, $k$ sire nested within breed, $j$ sex, $l$ dam, $m$ management, and $r$ animal at time $t$.

$$
\begin{aligned}
& \mu_{\mathrm{ijk} k m r t}=\alpha_{0}+\mathrm{b}_{0 \mathrm{i}}+\mathrm{s}_{0 \mathrm{j}} \\
& +\alpha_{1} \text { Age }_{\mathrm{t}}+\mathrm{b}_{1 \mathrm{i}} \text { Age }_{\mathrm{t}}+\mathrm{s}_{1 \mathrm{j}} \text { Age }_{\mathrm{t}} \\
& +\alpha_{2} \text { Age }^{2}+\mathrm{b}_{2 \mathrm{i}} \mathrm{Age}^{2}{ }_{\mathrm{t}}+\mathrm{s}_{2 \mathrm{j}} \mathrm{Age}^{2} \\
& +\alpha_{3} \text { Age }^{3}+b_{3 \mathrm{i}} \text { Age }^{3}+\mathrm{s}_{3 \mathrm{j}} \mathrm{Age}^{3}{ }_{\mathrm{t}}
\end{aligned}
$$


when $\mu_{\mathrm{ij} \text { klmrt }}$ is formed into a vector,

$$
\tau=\left[\alpha_{0} \alpha_{1} \alpha_{2} \alpha_{3} b_{01} b_{12} b_{21} b_{31} \ldots b_{0,97} b_{1,97} b_{2,97} b_{3,97} s_{02} s_{12} s_{22} s_{32}\right]
$$

The initial $b_{01} \ldots b_{31}$ and $s_{02} \ldots s_{32}$ are zero by constraint. The matrix $X$ is formed accordingly. The random effects $u$ consist of random cubic regression for sire, dam, management,

$$
\mathrm{u}=\left[\mathrm{u}_{1}^{\mathrm{T}}, \mathrm{u}^{\mathrm{T}}{ }_{2}, \mathrm{u}^{\mathrm{T}}{ }_{3}, \mathrm{u}^{\mathrm{T}}{ }_{4}\right]^{\mathrm{T}}
$$

where $u_{1}^{T}$ is a $388 \mathrm{X} 1$ vector of 97 sire random polynomial coefficients ( 4 for each sire). Thus,

$$
\mathrm{u}^{\mathrm{T}} \sim \mathrm{N}\left(0, \mathrm{G}_{1} \otimes \mathrm{I}_{97}\right)
$$

where $\mathrm{G}_{1}$ is a 4 x 4 -unstructured variance-covariance matrix. To provide an explicit model for the $u$ 's is possible. For example, for sire $k$ the effect is $\mathrm{u}_{01 \mathrm{k}}+\mathrm{u}_{11 \mathrm{k}} \mathrm{t}+\mathrm{u}_{21 \mathrm{k}} \operatorname{Age}_{\mathrm{t}}^{2}+\mathrm{u}_{31 \mathrm{k}} \operatorname{Age}_{\mathrm{t}}{ }_{\mathrm{t}}$. This structure is the same for dam, management and PE effects. Thus,

$$
\mathbf{u} \sim \mathrm{N}\left(\mathbf{0},\left[\begin{array}{cccc}
\mathbf{G}_{\text {Sire }} \otimes I_{97} & 0 & 0 & 0 \\
0 & \mathbf{G}_{\mathbf{M}} \otimes I_{581} & 0 & 0 \\
0 & 0 & \mathbf{G}_{\mathbf{M g}} \otimes I_{28} & 0 \\
0 & 0 & 0 & \mathbf{G}_{\mathbf{P E}} \otimes I_{1141}
\end{array}\right]\right)
$$

The matrix $\mathrm{Z}$ has the constant and $t, t^{2}, t^{3}$ values for each observation. Lastly, it is assumed that $\mathrm{R}=\sigma^{2}$ I. This may seem an unusual assumption for growth or repeated measures data. However, we are using the random regression to allow: a) for time-changing variances and b) correlation between successive times.

Growth data often exhibit increasing variance. A log-transformation has been used to stabilise the variance, but even on this scale, if variances change, the random regressions will attempt to accommodate that change in a quadratic fashion. Estimation of fixed effects and variance parameters is via residual per restricted maximum likelihood (Patterson and Thompson, 1971). Prediction of random effects is via best linear unbiased prediction (BLUP) (Robinson, 1991). The ASReml package is used for analysis. Gilmour et al. (1995) provided the details of the algorithms.

\section{Piecewise model}

In this study, a two-piece linear growth model was also employed to obtain the estimates of (co)variance components for pre- and post-weaning periods and the correlations between them. On the log scale, a preliminary analysis showed that quadratic terms were important for the piecewise model to fit the growth data. Suppose we wish to fit a piecewise quadratic model to mean $\log$-body weights, that is $\mathrm{E}(\ln ($ Body weight $))=$

$$
\begin{array}{ll}
\beta_{01}=\beta_{11} \mathrm{Age}+\beta_{21} \mathrm{Age}^{2}\left(\mathrm{Age} \leq \mathrm{Age}_{\text {Pre-weaning }}\right) & \text { (Equation 1) } \\
\beta_{02}=\beta_{12} \mathrm{Age}+\beta_{22} \mathrm{Age}^{2}\left(\mathrm{Age} \geq \mathrm{Age}_{\text {Pre-weaning }}\right) & \text { (Equation 2) }
\end{array}
$$


These two quadratics meet at pre-weaning age; Age $_{\text {Pre-weaning }}$ is the pre-weaning age in Equations 1 and 2.

Now, when Age $=$ Age $_{\text {Pre-weaning }}$,

$$
\beta_{01}+\beta_{11} \text { Age }_{\text {Pre-weaning }}+\beta_{21}\left(\text { Age }_{\text {Pre-weaning }}\right)^{2}=\beta_{02}+\beta_{12} \text { Age }_{\text {Pre-weaning }}+\beta_{22}\left(\text { Age }_{\text {Pre-weaning }}\right)^{2}
$$

We can solve for $\beta_{02}$ and hence, reduce the number of parameters. Clearly,

$$
\beta_{02}=\beta_{01}+\beta_{11}\left(\mathrm{Age}_{\text {Pre-weaning }}\right)-\beta_{12} \mathrm{Age}_{\text {Pre-weaning }}+\beta_{21} \mathrm{Age}_{\text {Pre-weaning }}^{2}-\beta_{22} \mathrm{Age}_{\text {Pre-weaning }}^{2}
$$

Thus, the model becomes $\mathrm{E}(\ln ($ Body weight $))=$

$$
\begin{aligned}
& \beta_{01}+\beta_{11} \operatorname{Age}+\beta_{21} \operatorname{Age}^{2}\left(\mathrm{Age} \leq \mathrm{Age}_{\text {Pre-weaning }}\right) \\
& \beta_{01}+\beta_{11} \mathrm{Age}_{\text {Pre-weaning }+} \beta_{12}\left(\mathrm{Age} \geq \mathrm{Age}^{2} \text { Pre-weaning }\right) \\
& \beta_{21} \mathrm{Age}^{2} \text { Pre-weaning }+\beta_{22}\left(\mathrm{Age}^{2} \leq \mathrm{Age}_{\text {Pre-weaning }}^{2}\right)\left(\mathrm{Age} \geq \mathrm{Age}_{\text {Pre-weaning }}\right)
\end{aligned}
$$

The model requires the definition of new explanatory variables to be used in the piecewise model. These variables are presented in Table 2.

Table 2. Partitioning age into pre- and post-weaning ages for linear and square calculations of age in piecewise model.

\begin{tabular}{lccrr}
\hline Age (days) & Pre-weaning (days) & Post-weaning (days) & (Pre-weaning age) $^{2}$ & $\left(^{\text {Post-weaning age })^{2}}\right.$ \\
\hline 0 & 0 & 0 & 10000 & 0 \\
100 & 100 & 0 & 62500 & 0 \\
$250^{\mathrm{a}}$ & 250 & 0 & 62500 & 0 \\
500 & 250 & 250 & 62500 & 187500 \\
700 & 250 & 450 & 427500 \\
\hline${ }^{a}$ Assumed weaning age for this example. (Post-weaning age) ${ }^{2}=\mathrm{Age}^{2}-$ Age $^{2}$ &
\end{tabular}

The same model building process discussed for the cubic model was used for the piecewise model. Therefore, the cubic random regression was replaced by the piecewise random regression formulation presented above.

The mixed model is $\mathrm{y}=\mathrm{X} \tau+\mathrm{Zu}+\mathrm{e}$

where $y$ is the vector of log-body weights for all animals at all measurement times, $\tau$ is the vector of fixed effects, $u$ is the vector of random effects, and $e$ is the vector of random errors. The matrices $\mathrm{X}$ and $\mathrm{Z}$ contain covariate values and indicators (factors) specific to each animal and sometimes to each measurement time. They extract the appropriate parts of $\tau$ and $u$ for each animal and time. The model fitted using ASREML (Gilmour et al., 2000), included fixed effects of sex, sire breed, age (linear, quadratic), as well as two-way interactions between the age parameters and sex or breed. Fixed effects in the model were tested using a Wald $F$-statistic (ASReml $F$ values) obtained by dividing the Wald statistic by its degree of freedom.

The piecewise model (sire) in this study assumes that the growth curve of each individual animal follows a second-degree polynomial. The quadratic coefficient was estimated within the breed and sex effect to take into account differences in growth due to breed and sex. This model was specified as a function of five growth parameters; mean linear and quadratic effects of pre-weaning and post-weaning growth as a covariate. 


\section{RESULTS}

Log-likelihood, number of parameters, observations, fixed effects, and error variances for the two models are shown in Table 3. The residual variation of the piecewise model was lower than that of the cubic model. The random effects significantly reduced the residual variation for both models (Table 3).

\begin{tabular}{lll}
\multicolumn{2}{l}{ Table 3. Summary descriptions of the cubic and piecewise models. } & \\
\hline & Cubic & Piecewise \\
\hline Log-likelihood (fixed + random effects) & 20722.8 & 21119.6 \\
Log-likelihood (fixed effects) & 16004.5 & 16287.6 \\
Number of model parameters & 22 & 32 \\
Number of observations & 11936 & 11936 \\
Number of fixed effects & 12 & 15 \\
Error variance & 0.00833 & 0.00753 \\
\hline
\end{tabular}

aNumber of variance components.

Table 4 shows the ASReml $F$ values (Wald $F$-statistic) to test the significance of fixed effects considered in the models. In the cubic model, differences due to breed group were significant $(\mathrm{P}<0.01)$. The third order of age and breed by $\mathrm{Age}^{3}$ interactions as well as sex by $\mathrm{Age}^{3}$ interactions were significant $(\mathrm{P}<0.01)$, indicating the importance of cubic form of the growth model (Table 4). As it has shown, in the piecewise model differences due to breed and sex and their interactions with second order of time were significant (Table 4).

Table 4. ASReml $F$ value to test the fixed effects in cubic and piecewise models.

\begin{tabular}{|c|c|c|c|c|c|}
\hline \multicolumn{3}{|c|}{ Piecewise model } & \multicolumn{3}{|c|}{ Cubic model } \\
\hline Fixed effects & d.f. & $F$ value & Fixed effects & d.f. & $F$ value \\
\hline Mean & 1 & 162682.1 & Mean & 1 & 176183.87 \\
\hline Breed & 6 & 42.99 & Breed & 6 & 42.78 \\
\hline Sex & 7 & 158.26 & Sex & 1 & 145.89 \\
\hline Age $_{\text {pre }}$ & 1 & 24393.01 & Age & 1 & 17513.72 \\
\hline $\mathrm{Age}_{\text {prest }}^{\text {pre }}$ & 1 & 3043.45 & $\mathrm{Age}^{2}$ & 1 & 1056.80 \\
\hline $\mathrm{Age}^{2}{ }_{\text {pre }}^{\text {pos }}$ & 1 & 502.3 & $\mathrm{Age}^{3}$ & 1 & 1555.57 \\
\hline $\mathrm{Age}^{2^{\text {post }}}$ & 1 & 100.73 & Breed.Age & 6 & 11.41 \\
\hline Breed.Age & 6 & 1.32 & Breed.Age $^{2}$ & 6 & 8.31 \\
\hline Breed.Age ${ }_{\text {post }}$ & 6 & 9.74 & Breed.Age ${ }^{3}$ & 6 & 6.52 \\
\hline Breed.Age ${ }^{2}$ pst & 6 & 1.83 & Sex.Age & 1 & 23.85 \\
\hline Breed.Age ${ }^{\text {pre }}$ & 6 & 15.89 & Sex.Age ${ }^{2}$ & 1 & 4.57 \\
\hline Sex.Age ${ }_{\text {pre }}$ & 7 & 1.32 & Sex.Age ${ }^{3}$ & 1 & 3.57 \\
\hline Sex.Age & 7 & 5.5 & & & \\
\hline Sex.Age ${ }^{2}$ pre & 7 & 226.38 & & & \\
\hline Sex.Age ${ }^{2}{ }_{\text {post }}^{\text {pre }}$ & 7 & 7.58 & & & \\
\hline
\end{tabular}

\section{Growth paths}

The weight of an average animal at any time for the combination of fixed effects was obtained from the solution of model to plot average growth path and average deviation of crossbred cattle from purebred Hereford. The growth paths (estimated monthly body weights) from birth to slaughter for seven breeds are displayed in Figures 1 and 2. In both models, body weights of all breeds increased over pre-weaning period, held fairly steady (slightly flatten- 
ing) over the dry season then went up again toward the end of the feedlot period during June to October (wet season).

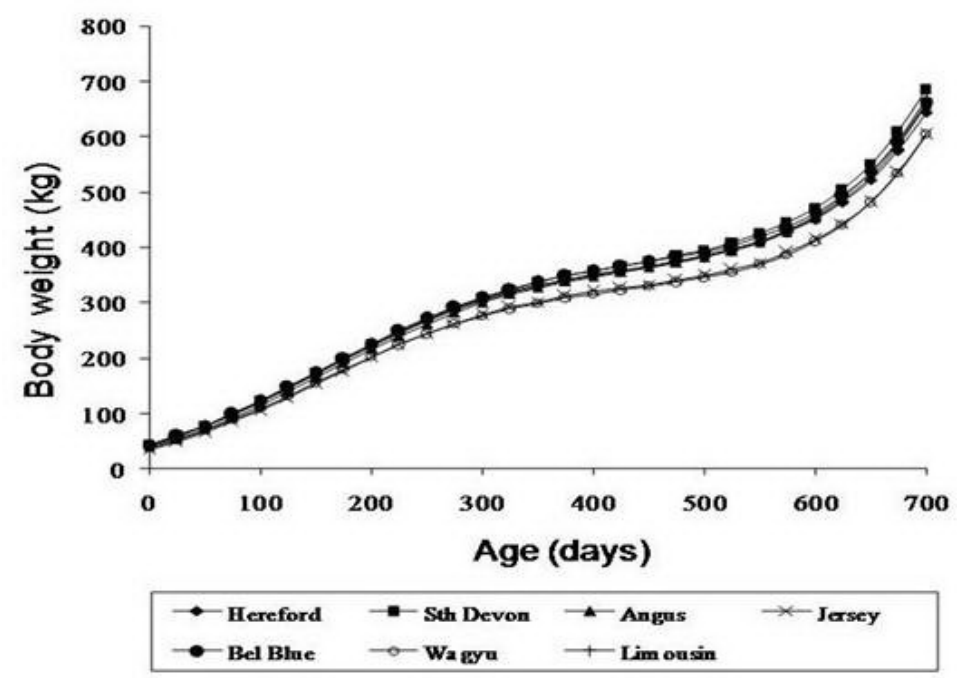

Figure 1. Growth paths (birth to slaughter) for seven crossbreeds derived from the cubic growth model.

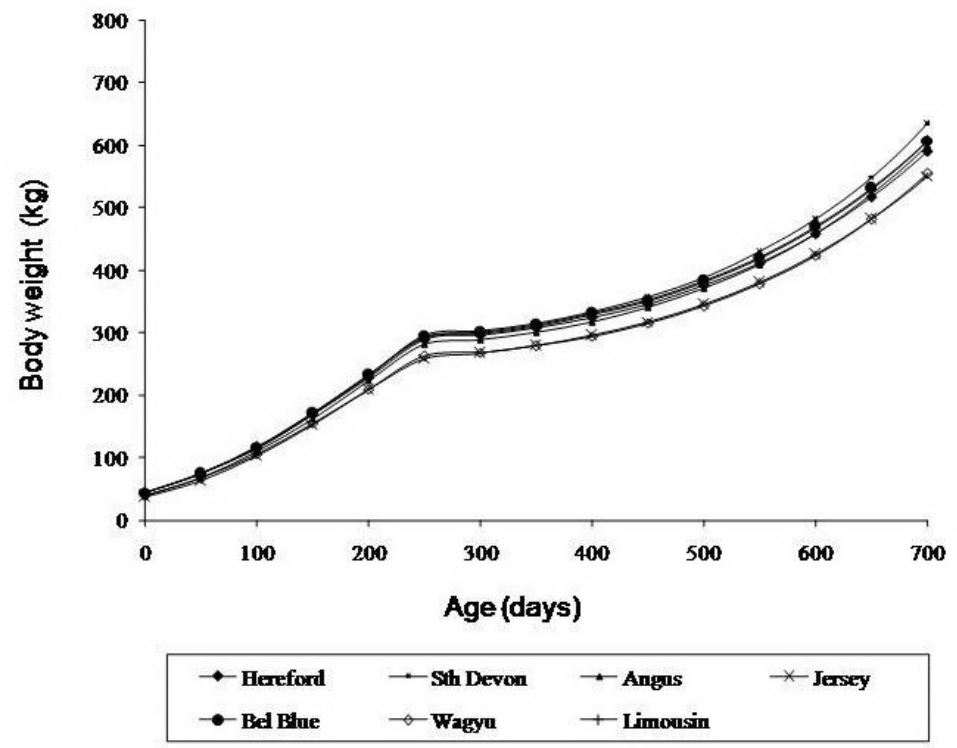

Figure 2. Growth paths (birth to slaughter) for seven crossbreeds derived from the piecewise growth model.

\section{Crossbred comparisons with purebred Hereford}

Figures 3 and 4 illustrate the percentage deviation of estimated average weight of 
crossbreds from purebred Herefords at various ages obtained from the cubic and piecewise models, indicating very similar pattern for both models. Although the magnitude of the percentage of deviation among breeds was variable over time, South Devon, Belgian Blue and Limousin calves were consistently heavier and Jersey and Wagyu were lighter than Hereford calves, as expected. Basically, breed differences were consistent across ages except that Jersey and Angus were relatively lighter in the first 200 days than 200-700 days. The magnitude of the percentage of deviation during pre-weaning period was approximately -20 to $-10 \%$, and -12 to $-8 \%$ for Jersey and Wagyu, respectively. After weaning they remained steady at about $-10 \%$; however, the magnitude of the percentage of deviation for Jersey became smaller than Wagyu (Figures 3 and 4). During pre-weaning, an interesting pattern was shown for Angus, where its percent of deviation increased dramatically, clearly lighter than the Hereford calves. Obviously, after weaning the direction of the deviation changed, so Angus calves became heavier than Hereford calves. Breed ranking at the post-weaning period was the same as the pre-weaning period (Figures 3 and 4).

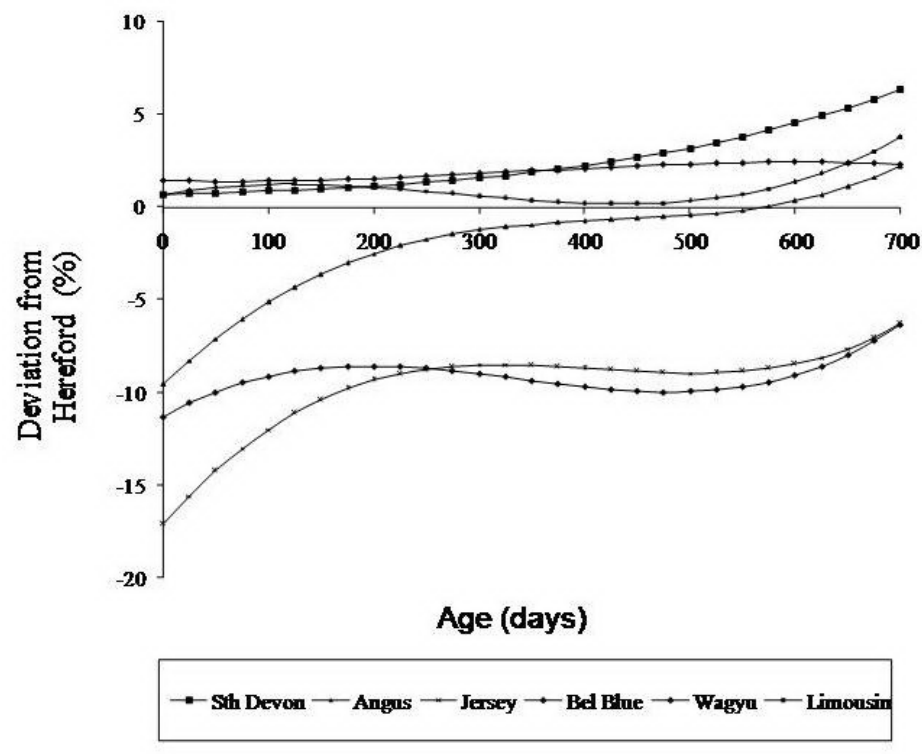

Figure 3. Deviation of estimated body weight of six crossbreds from purebred Hereford derived from the cubic model.

\section{(Co)variance components}

Cubic random coefficient models were postulated for sire, dam, permanent environmental, and management effects. In many cases the data did not support inclusion of all terms because the model failed to converge in estimations. Table 5 presents the (co)variance components that were able to be fitted. Estimated components that are marked as blank were estimated to be on the boundary; that is they converged to zero. A quadratic random effect for sire and dam were in the model, but had to be removed because the algorithm failed to converge. The covariance between sire constant and linear was not able to be estimated (Table 5). Finally, 22 (co)variance components were able to be estimated (Table 5). 


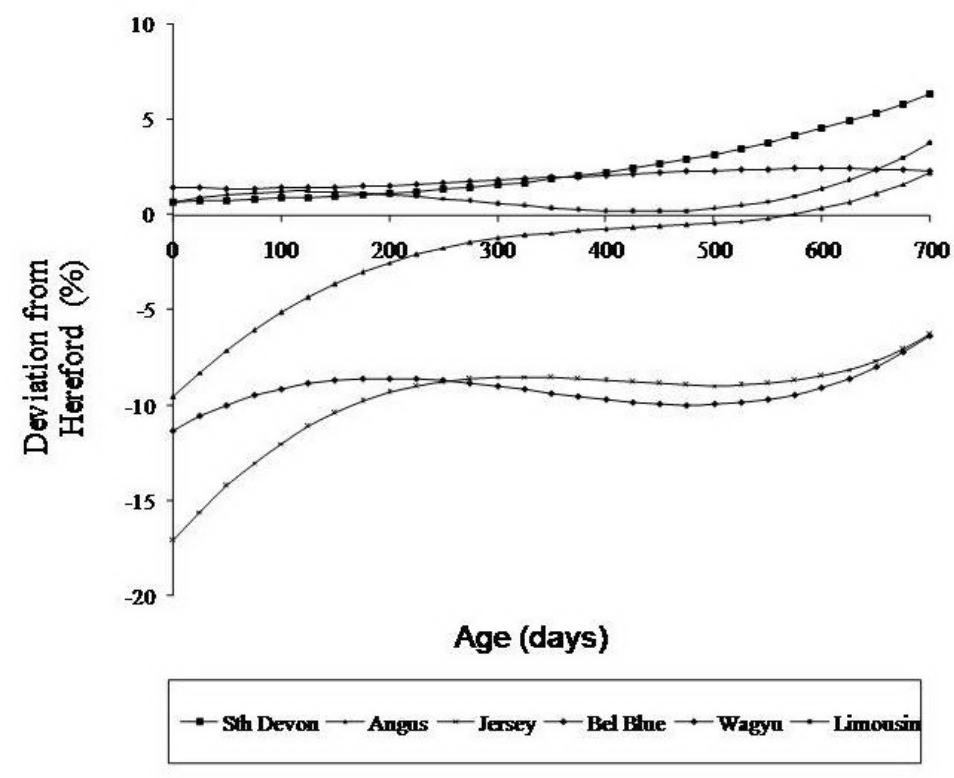

Figure 4. Deviation of estimated body weight of six crossbreds from purebred Hereford derived from the piecewise model.

Table 5. Estimated variance components (on diagonal) and covariances (off diagonal) from the cubic sire model.

\begin{tabular}{|c|c|c|c|c|c|c|c|c|c|c|c|c|}
\hline & 1 & 2 & 3 & 4 & 5 & 6 & 7 & 8 & 9 & 10 & 11 & 12 \\
\hline 1. Sire constant & $\checkmark$ & & & & & & & & & & & \\
\hline 2. Sire linear & & $\checkmark$ & & & & & & & & & & \\
\hline 3. Maternal constant & & & $\checkmark$ & & & & & & & & & \\
\hline 4. Maternal linear & & & $\checkmark$ & $\checkmark$ & & & & & & & & \\
\hline 5. Management.constant & & & & & $\checkmark$ & & & & & & & \\
\hline 6. Management.linear & & & & & $\checkmark$ & $\checkmark$ & & & & & & \\
\hline 7. Management.quadratic & & & & & $\checkmark$ & $\checkmark$ & $\checkmark$ & & & & & \\
\hline 8. Management.cubic & & & & & $\checkmark$ & $\checkmark$ & $\checkmark$ & $\checkmark$ & & & & \\
\hline 9. PE.constant & & & & & & & & & $\checkmark$ & & & \\
\hline 10. PE.linear & & & & & & & & & $\checkmark$ & $\checkmark$ & & \\
\hline 11. PE.quadratic & & & & & & & & & $\checkmark$ & $\checkmark$ & $\checkmark$ & \\
\hline 12. Residual & & & & & & & & & & & & $\checkmark$ \\
\hline
\end{tabular}

Many (32) (co)variances were able to be estimated for the piecewise sire model. Due to small variances for the quadratic terms of sire, maternal and permanent environmental effects, their (co)variances with mean and linear terms were not able to be estimated (Table 6).

Figure 5 shows the relative contribution of genetic and non-genetic variance components to the total phenotypic variance obtained from the two growth models. The variance bars range between zero and 98 (Figure 5). Generally, 79-93\% of the variability was non-genetic for the cubic and piecewise models, respectively. The management variance contributed up to $68-71 \%$ of the phenotypic variance of body weights for the cubic and piecewise models, respectively. The genetic variation ranges from zero (for piecewise) to $1 \%$ (for cubic) of total variation (Figure 5). 
Table 6. Estimated variance (on diagonal) and covariance (off diagonal) components from the sire piecewise model.

\begin{tabular}{|c|c|c|c|c|c|c|c|c|c|c|c|c|c|c|c|}
\hline & 1 & 2 & 3 & 4 & 5 & 6 & 7 & 8 & 9 & 10 & 11 & 12 & 13 & 14 & 15 \\
\hline 1. Sire.Age & $\checkmark$ & & & & & & & & & & & & & & \\
\hline 2. Sire.Age & $\checkmark$ & $\checkmark$ & & & & & & & & & & & & & \\
\hline 3. Sire.Age $\mathrm{P}^{\text {Pre-weaning }}$ & $\checkmark$ & $\checkmark$ & $\checkmark$ & & & & & & & & & & & & \\
\hline 4. Maternal.Ágenenang & & & & $\checkmark$ & & & & & & & & & & & \\
\hline 5. Maternal.Age & & & & $\checkmark$ & $\checkmark$ & & & & & & & & & & \\
\hline 6. Maternal.Age ${ }^{\text {Pre-weaning }}$ & & & & $\checkmark$ & $\checkmark$ & $\checkmark$ & & & & & & & & & \\
\hline 7. Management.Age & & & & & & & $\checkmark$ & & & & & & & & \\
\hline 8. Manag.Age & & & & & & & $\checkmark$ & $\checkmark$ & & & & & & & \\
\hline 9. Manag.Age $\mathrm{P}^{\text {Pre-weaning }}$ & & & & & & & $\checkmark$ & $\checkmark$ & $\checkmark$ & & & & & & \\
\hline 10. Manag.Age ${ }^{\text {Post-weaning }}$ & & & & & & & $\checkmark$ & $\checkmark$ & $\checkmark$ & $\checkmark$ & & & & & \\
\hline 11. Manag.Age ${ }^{\text {Pre-veraning }}$ & & & & & & & $\checkmark$ & $\checkmark$ & $\checkmark$ & $\checkmark$ & $\checkmark$ & & & & \\
\hline 12. PE.Age & & & & & & & & & & & & $\checkmark$ & & & \\
\hline 13. PE.Age & & & & & & & & & & & & $\checkmark$ & $\checkmark$ & & \\
\hline 14. PE.Age ${ }^{\text {Pre-weaning }}$ & & & & & & & & & & & & & & $\checkmark$ & \\
\hline 15. Overall restryacinuag & & & & & & & & & & & & & & & $\checkmark$ \\
\hline
\end{tabular}

Manag = management; PE $=$ permanent environmental. Tick marks indicate the components that were able to be estimated.

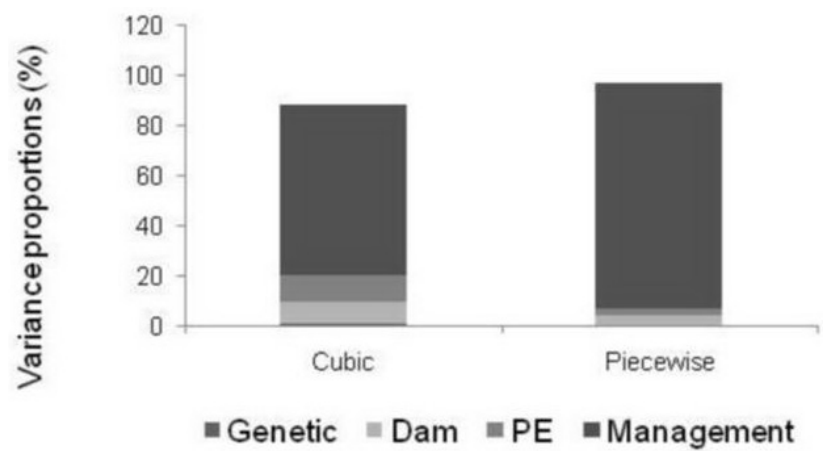

Figure 5. Proportions of variance components obtained from cubic and piecewise models. PE $=$ permanent environment.

Plotting residuals versus fitted values of both models produced a distribution of points scattered about zero (Figures 6 and 7). For both models the fit around weaning age ( 200-250 days) indicates predominantly positive residuals.

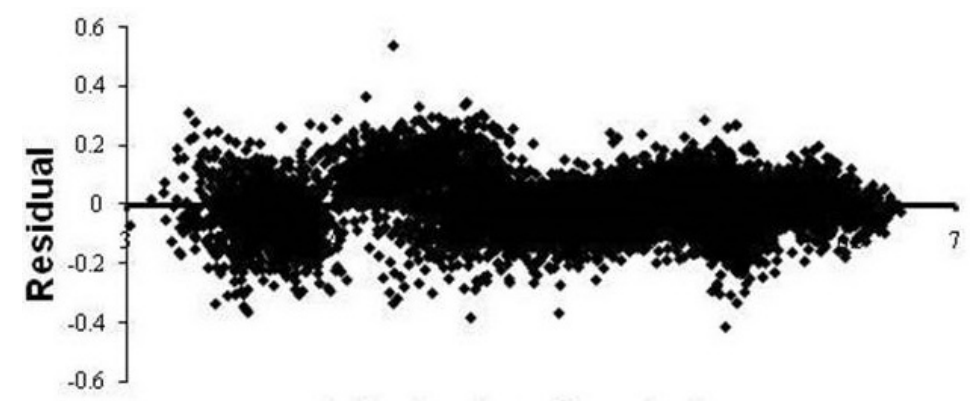

Fitted values (Log-kg)

Figure 6. Plot of residual $v s$ fitted values for the piecewise model. 


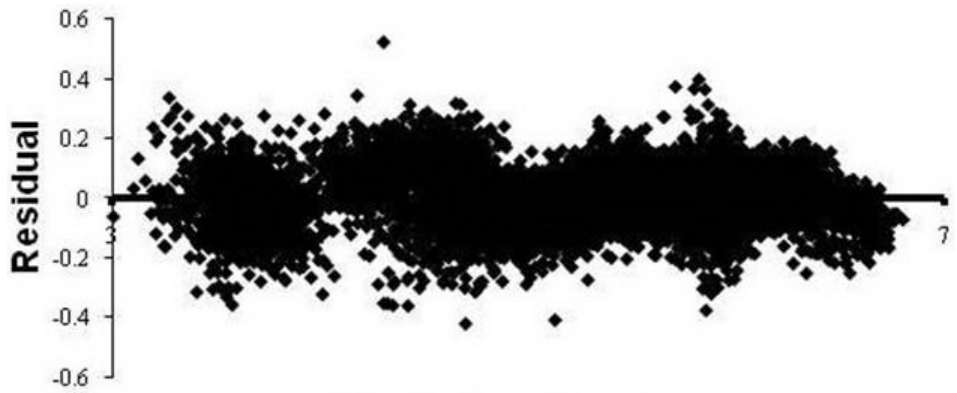

Fitted values (Log-kg)

Figure 7. Plot of residual $v s$ fitted values for the cubic model.

\section{DISCUSSION}

As stated earlier, the aims of this paper were to provide a view of the statistical and then biological aspects of the cubic and the piecewise random regression analyses; as a means for dividing successive ages into meaningful segments, and capturing key features of change in each segment. It is known that growth is continuous during an animal's life and is evaluated by growth rate or by weight and size increases during different stages of the growth path, for example pre- and post-weaning and feedlot periods. Body weights change with age and there is evidence that these changes are influenced by genetic and non-genetic factors (Atchley et al., 1997). From an animal modelling point of view, interest lies in genetic and non-genetic parameters that describe the change of such traits over time (Meyer, 2004; Williams et al., 2009).

In this study, random regression analysis was employed to provide a method for analyzing independent components of variation that reveal specific patterns of change over time. Recently, random regression models have been advocated to fit growth data (Schenkel et al., 2002; Hassen et al., 2003). Also, the method was applied by Varona et al. (1997) and Arango et al. (2002) on the weights of mature beef cows.

When comparing cubic and piecewise models, both of them were very similar in their fit to the data; with the piecewise model being marginally better. In addition, it can be seen that the piecewise model estimates more parameters than the cubic model (32 versus 22). The loglikelihood of piecewise model was greater than the cubic model. Also, the residual variance of the piecewise model was lower than that of the cubic model. Moreover, the plots of residual versus fitted value show that a piecewise model performs better than a cubic model at the end of the trajectory for higher values on these data. For both models the fit around weaning age ( 200-250 days) was not very good with predominantly positive residuals. After using random regression with a polynomial, the cubic model was subject to overestimates at the end of the trajectory, in particular beyond 650 days. That occurred because the variances associated with ages of most missing records become erratic. Further, most models assume that the residuals are distributed normally and independent, with zero mean and equal variance, but in practice a systematic pattern was observed in the residuals over the growth trajectory because the mean profile was not adequately modelled (Figures 6 and 7). Moreover, it is possible that the fit is worst for traits with the smallest variances. This remains an unresolved problem for modelling that utilizes polynomials as has been shown by others (Meyer, 2005a). Several strategies may 
be used for obtaining "better" estimates. One could be to use larger, more carefully selected data. Another strategy would be to use functions other than polynomials that are less susceptible to artifacts (Aggrey, 2002; Meyer, 2005b; Bohmanova et al., 2008). Although the choice of which type of function to use might not have a large effect on the parameter estimates within the interval that data were collected, the function might be more important as soon as data are extrapolated (Kirkpatrick et al., 1990; Aggrey, 2009; Vitezica et al., 2010).

Overall, it is suggested that investigations into alternative models would seem to be important and perhaps modelling heifers and steers separately might be advantageous. Steers seem to have three phases of growth and a piecewise linear model of three pieces may in fact result in a better fit. However, preliminary analysis found that this was poor for heifers because the middle piece (weaning, pre-feedlot) had too few (sometimes zero!) weight measurements to allow accurate estimates. It may have been useful for steer data.

The results of relative contribution of variance components from both cubic and piecewise growth models in this study indicated that non-genetic variation accounted for the larger proportion of the total variance. Also, the management variation contributed to the largest proportion of non-genetic and total variation of body weights as nutrition was based on the pasture. The seasonal pattern (growth curves) of green herbage mass and senesced residues (dead and litter) at Wandilo and Struan demonstrated that the dry matter of available green herbage mass and senesced residues (dead and litter) increased over June to October (GrassGro, 2002). It also exhibited declining levels of green herbage mass (but high levels of senesced residues) in spring and low levels of green herbage mass in summer through autumn, i.e., the approximate period between 250 days (December) to 350 days (March), which corresponded with the dry season. For the period March-April total pasture availability still decreased (GrassGro, 2002). Also, based on the seasonal pattern of in vitro digestibility and energy content for the green and senesced fractions of herbage mass at Wandilo and Struan, the digestibility of green herbage mass progressively declined in summer through autumn from 0.77 to 0.58 , remained relatively constant over autumn at $0.57-0.60$ and was uniformly high in winter and spring at 0.75 to 0.78 . The digestibility of the senesced fraction remained in the range $0.45-0.55$ digestibilities (GrassGro, 2002). Therefore, the pattern of growth curve illustrates occurrence of a summer-autumn feed gap due to the sub-optimal nutritive value of green herbage mass from late spring through summer-early autumn. Thus, the seasonal pattern of quantity and quality of pastures at Struan and Wandillo indicated that the limitations of the feed year include a feed gap in summer-early autumn due to low herbage mass associated with dry season, and a feed gap in summer-autumn associated with only moderate pasture quality (digestibility) of secondary re-growth pasture (GrassGro, 2002). The higher gains of calves on pasture can be due to the higher availability of nutrients for their dams, particularly at the start of the grazing season and if they had been undernourished during backgrounding as part of the post-weaning period. The decrease in the rate of growth after weaning during the dry period was primarily due to decreasing quality of pasture as the season progressed. Thus, the significance of these feed gaps for the post-weaning growth of steers and heifers was highlighted through the backgrounding period. Similar growth patterns were observed for both cubic and piecewise models throughout pre-weaning and post-weaning periods (Figures 1 and 2). However, during the post-weaning period, two groups of sires, heavy and small, showed different growth patterns.

Breed differences in performance characteristics are an important genetic resource for improving efficiency of beef production. Diverse breeds are required to exploit heterosis and 
complementarity through crossbreeding to match genetic potential with diverse markets, feed resources and climates. The percent deviation of estimated average weight of crossbreds from purebred Herefords from birth to slaughter obtained from both models indicated that, as might be expected, the South Devon, Belgian Blue and Limousin calves were consistently heavier and Angus Jersey and Wagyu were lighter than Hereford calves. Jersey and Angus demonstrated the best combination of minimum birth weight and maximum growth rate over time.

\section{REFERENCES}

Aggrey SE (2002). Comparison of three nonlinear and spline regression models for describing chicken growth curves. Poult. Sci. 81: 1782-1788.

Aggrey SE (2009). Logistic nonlinear mixed effects model for estimating growth parameters. Poult. Sci. 88: 276-280.

Arango JA, Cundiff LV and Van Vleck LD (2002). Breed comparisons of Angus, Charolais, Hereford, Jersey, Limousin, Simmental, and South Devon for weight, weight adjusted for body condition score, height, and body condition score of cows. J. Anim. Sci. 80: 3123-3132.

Atchley WR, Xu S and Cowley DE (1997). Altering developmental trajectories in mice by restricted index selection. Genetics 146: 629-640.

Bohmanova J, Miglior F, Jamrozik J, Misztal I, et al. (2008). Comparison of random regression models with Legendre polynomials and linear splines for production traits and somatic cell score of Canadian Holstein cows. J. Dairy Sci. 91: 3627-3638.

Brown JE, Fitzhugh HA Jr and Cartwright TC (1976). A comparison of nonlinear models for describing weight-age relationships in cattle. J. Anim. Sci. 42: 810-818.

Fitzhugh HA Jr (1976). Analysis of growth curves and strategies for altering their shape. J. Anim. Sci. 42: 1036-1051.

Forni S, Piles M, Blasco A, Varona L, et al. (2009). Comparison of different nonlinear functions to describe Nelore cattle growth. J. Anim. Sci. 87: 496-506.

Gilmour AR, Thompson R and Cullis BR (1995). Average information reml: An efficient algorithm for variance parameter estimation in linear mixed models. Biometrics 51: 1440-1450.

Gilmour AR, Bulls BR, Welham SJ and Thompson R (2000). Asreml Reference Manual, NSW Agriculture Biometric Bulletin no. 3. Orange Agricultural Institute, Orange.

GrassGro (2002). Analysing pasture and animal production. Version 2.4.1. Horizon Agriculture Pty Limited, New South Wales.

Hassen A, Wilson DE and Rouse GH (2003). Estimation of genetic parameters for ultrasound-predicted percentage of intramuscular fat in Angus cattle using random regression models. J. Anim. Sci. 81: 35-45.

Hodson DJ (1966). Fitting segmented curves whose join points have to be estimated. Am. Stat. Assoc. 61: 1097-1125.

Kirkpatrick M, Lofsvold D and Bulmer M (1990). Analysis of the inheritance, selection and evolution of growth trajectories. Genetics 124: 979-993.

Meyer K (2004). Scope for a random regression model in genetic evaluation of beef cattle for growth. Livest. Prod. Sci. 86: 69-83.

Meyer K (2005a). Advances in methodology for random regression analyses. Aust. J. Exp. Agric. 45: 847-858.

Meyer K (2005b). Random regression analyses using B-splines to model growth of Australian Angus cattle. Genet. Sel. Evol. 37: 473-500.

Patterson HD and Thompson R (1971). Recovery of inter-block information when block sizes are unequal. Biometrika 58: 545-554.

Pitchford WS, Mirzaei HR, Deland MPB, Afolayan RA, et al. (2006). Variance components for birth and carcass traits of crossbred cattle. Aust. J. Exp. Agric. 46: 225-231.

Robinson GK (1991). That blup is a good thing: the estimation of random effects. Stat. Sci. 6: 15-32.

Schenkel FS, Miller SP, Jamrozik J and Wilton JW (2002). Two-step and random regression analyses of weight gain of station-tested beef bulls. J. Anim. Sci. 80: 1497-1507.

Schinckel AP, Adeola O and Einstein ME (2005). Evaluation of alternative nonlinear mixed effects models of duck growth. Poult. Sci. 84: 256-264.

Speidel SE, Enns RM and Crews DH Jr (2010). Genetic analysis of longitudinal data in beef cattle: a review. Genet. Mol. Res. 9: 19-33.

Varona L, Moreno C, García Cortes LA and Altarriba J (1997). Multiple trait genetic analysis of underlying biological variables of production functions. Livest. Prod. Sci. 47: 201-209.

Vitezica ZG, Marie-Etancelin C, Bernadet MD, Fernandez X, et al. (2010). Comparison of nonlinear and spline regression

Genetics and Molecular Research 10 (3): 2230-2244 (2011)

CFUNPEC-RP www.funpecrp.com.br 
models for describing mule duck growth curves. Poult. Sci. 89: 1778-1784.

Warren JH, Schalles RR and Milliken G (1980). A segmented line regression procedure to describe growth in beef cows. Growth 44: 160-166.

Williams JL, Garrick DJ and Speidel SE (2009). Reducing bias in maintenance energy expected progeny difference by accounting for selection on weaning and yearling weights. J. Anim. Sci. 87: 1628-1637. 